\title{
Widely Linear Modeling for Frequency Estimation in Unbalanced Three-Phase Power Systems
}

\author{
Dahir H. Dini, Student Member, IEEE, and Danilo P. Mandic, Senior Member, IEEE
}

\begin{abstract}
Real-time frequency estimation in three-phase power systems is revisited from the state space point of view, in order to provide a unified framework for frequency tracking in both balanced and unbalanced system conditions. This is achieved by using a novel class of widely linear complex valued Kalman filters, which provide unbiased frequency estimation and are faster converging and more robust to noise and harmonic artifacts than the existing methods. It is shown that the Clarke's transformed threephase voltage is circular for balanced systems and noncircular for unbalanced ones, making the proposed widely linear estimation perfectly suited both to identify the fault and to provide accurate estimation in unbalanced conditions, critical issues where standard models typically fail. The analysis and simulations show that the proposed model outperforms the recently introduced widely linear stochastic gradient-based frequency estimators, based on the augmented complex least mean square. Comprehensive simulations on synthetic and real-world power system data, in both balanced and unbalanced conditions, support the approach.
\end{abstract}

Index Terms-Augmented complex Kalman filter (ACKF), complex circularity, frequency estimation, smart grid, widely linear estimation.

\section{INTRODUCTION}

$\mathbf{T}$ HE FREQUENCY of a power system is a crucial power quality parameter and is allowed to vary around its nominal value only within a prescribed tolerance level. Large frequency deviations are harmful to the system and arise in the presence of unbalanced system conditions, such as generation-consumption imbalances or unexpected conditions which require corrective actions. With the emergence of smart grids, system stability issues become even more pronounced, owing to more and more diversified generation and increasingly unpredictable power consumption. Frequency tracking and estimation in the context of smart grid is a key parameter both for the protection of power system and for improved power quality; for instance, frequent switching from the main grid to microgrids and electricity islands and dual natures of some loads require rapid frequency trackers to trigger corrective actions to maintain power quality.

A number of approaches for frequency tracking have been proposed, including least mean square (LMS) adaptive filters [1], state space algorithms based on Kalman filters [2], [3], and

Manuscript received January 25, 2012; revised July 17, 2012; accepted July 19, 2012. Date of publication November 19, 2012; date of current version December 29, 2012. The Associate Editor coordinating the review process for this paper was Dr. Carlo Muscas.

The authors are with the Department of Electrical and Electronic Engineering, Imperial College London, London SW7 2AZ, U.K. (e-mail: dahir.dini@ ic.ac.uk; d.mandic@ic.ac.uk).

Color versions of one or more of the figures in this paper are available online at http://ieeexplore.ieee.org.

Digital Object Identifier 10.1109/TIM.2012.2214953
Fourier transform-based approaches [4], [5]. However, these techniques either are only optimal for balanced systems (e.g., systems with line voltages of equal amplitudes) or are designed specifically for single-phase systems [6]-[8]; these cannot fully characterize three-phase power systems where the line-to-line voltages also need to be taken into account.

In the future smart grid, the system frequency will undergo deviations due to the following: imbalance in generation $(\mathrm{G})$ and load (L) (rise for $G>L$ and decay for $G<L$ ), single and dual phase faults or sags (Clarke's transformed unbalanced voltage gives wrong frequency reading), dynamical loads and dual character of $\mathrm{G}$ and $\mathrm{L}$, and a number of issues causing harmonics and transient stability issues (nonlinear loads and reactive power compensation). Accurate frequency estimators are a prerequisite for fault identification and troubleshooting, highlighting the need for a unified frequency estimation framework in three-phase power systems with the following characteristics:

1) robust to measurement noise and harmonics in the system, including the slowly floating ones which are not integer multiples of system frequency;

2) real-time adaptive, fast converging, and asymptotically unbiased;

3) minimum variance and statistically consistent, i.e., approaching theoretical performance bounds;

4) capable of catering for both balanced and unbalanced systems under the same umbrella and, at the same time, tracking frequency and identifying system disturbance.

To deal with the three-phase voltages simultaneously, standard frequency trackers employ Clarke's $\alpha \beta$ transformation which maps the three-phase voltages onto the variables $v_{0}, v_{\alpha}$, and $v_{\beta}$ to produce the complex signal, $v=v_{\alpha}+j v_{\beta}$, with $v_{0}$ vanishing for a balanced system [3]. However, current strictly linear estimators are not capable of capturing full second-order information for unbalanced voltage conditions, resulting in an oscillatory estimation error at twice the system frequency [9]. Our recent work [10] establishes that, for unbalanced systems, the $\alpha \beta$ voltage is noncircular, and widely linear models are required for accurate system representation, achieved based on the augmented complex LMS (CLMS) (ACLMS) [11].

The ACLMS framework is shown to cater for both balanced and unbalanced conditions. However, it assumes that the measured voltages are noise free; otherwise, the frequency estimates are inaccurate. This assumption is not practical, as real-world power systems are typically corrupted by harmonics and random noise sources. To this end, we extend the gradientbased widely linear frequency estimation framework in [10] 
and introduce a class of widely linear (augmented) Kalman filters. Owing to the underpinning state space representation, this approach offers enhanced accuracy and faster convergence, together with robustness to noise and harmonics. Illustrative simulations on unbalanced and noisy real-world power systems support the analysis.

\section{BACKGROUND}

\section{A. Augmented Complex Statistics and Widely Linear Modeling}

Prior to introducing an optimal linear estimator for the generality of complex signals, consider the real valued mean square estimator (MSE) of a random vector $y$ in terms of a real observation $\mathbf{x}$, i.e., $\hat{\mathbf{y}}=E\{\mathbf{y} \mid \mathbf{x}\}$. For zero-mean jointly normal $\mathbf{y}$ and $\mathbf{x}$, the optimal estimator is linear, i.e.,

$$
\hat{\mathbf{y}}=\mathbf{H x}
$$

where $\mathbf{H}$ is a coefficient matrix. Standard "strictly linear" estimation in the complex domain $(\mathbb{C})$ assumes the same model, but with complex valued $\mathbf{y}, \mathbf{x}$, and $\mathbf{H}$, and is widely used. However, observe that both the real $\mathbf{y}_{r}$ and imaginary $\mathbf{y}_{i}$ parts of the vector $\mathbf{y}$ are real valued, and thus

$$
\hat{\mathbf{y}}_{r}=E\left\{\mathbf{y}_{r} \mid \mathbf{x}_{r}, \mathbf{x}_{i}\right\} \quad \hat{\mathbf{y}}_{i}=E\left\{\mathbf{y}_{i} \mid \mathbf{x}_{r}, \mathbf{x}_{i}\right\} .
$$

Substituting $\mathbf{x}_{r}=\left(\mathbf{x}+\mathbf{x}^{*}\right) / 2$ and $\mathbf{x}_{i}=\left(\mathbf{x}-\mathbf{x}^{*}\right) / 2 \jmath$ yields

$$
\hat{\mathbf{y}}_{r}=E\left\{\mathbf{y}_{r} \mid \mathbf{x}, \mathbf{x}^{*}\right\} \quad \hat{\mathbf{y}}_{i}=E\left\{\mathbf{y}_{i} \mid \mathbf{x}, \mathbf{x}^{*}\right\}
$$

where $(\cdot)^{*}$ is the complex-conjugate operator, and using (1), we obtain the widely linear complex estimator ${ }^{1}$ [12]

$$
\mathbf{y}=\mathbf{H x}+\mathbf{G} \mathbf{x}^{*}=\mathbf{W} \mathbf{x}^{a} .
$$

The matrix $\mathbf{W}$ comprises the coefficient matrices $\mathbf{H}$ and $\mathbf{G}$, and $\mathbf{x}^{a}=\left[\mathbf{x}^{T}, \mathbf{x}^{H}\right]^{T}$ is the "augmented" input vector, where $[\cdot]^{T}$ and $[\cdot]^{H}$ are the transpose and complex conjugate-transpose operators, respectively. The full second-order information is contained in the augmented covariance matrix

$$
\mathbf{R}_{\mathbf{x}}^{a}=E\left\{\mathbf{x}^{a} \mathbf{x}^{a H}\right\}=\left[\begin{array}{ll}
\mathbf{R}_{\mathbf{x}} & \mathbf{P}_{\mathbf{x}} \\
\mathbf{P}_{\mathbf{x}}^{*} & \mathbf{R}_{\mathbf{x}}^{*}
\end{array}\right]
$$

which encompasses both the covariance $\mathbf{R}_{\mathbf{x}}=E\left\{\mathbf{x x}^{H}\right\}$ and pseudocovariance $\mathbf{P}_{\mathbf{x}}=E\left\{\mathbf{x x}^{T}\right\}$. Complex signals with vanishing pseudocovariances, i.e., $\mathbf{P}_{\mathbf{x}}=\mathbf{0}$, are termed second order circular (proper) and are characterized by rotation invariant probability distributions; otherwise, the signals are noncircular (improper). For more details about widely linear modeling, see [11].

\section{B. Improperness and WLAR Modeling}

The so-called "proper" data have equal powers in the real and imaginary parts, while for the improper data, the covariance

\footnotetext{
${ }^{1}$ The "widely linear" model is associated with the signal generating system, whereas "augmented statistics" describe statistical properties of measured signals. Both the terms "widely linear" and "augmented" are used to name the resulting algorithms-in our work, we mostly use the term "augmented."
}

is greater than the absolute pseudocovariance. This can be illustrated from the expression for the pseudocovariance

$$
E\left\{z z^{T}\right\}=E\left\{\left(x^{2}-y^{2}\right)+2 \jmath x y\right\}=\sigma_{x}^{2}-\sigma_{y}^{2}+2 \jmath \rho_{x y}
$$

where the symbol $\sigma^{2}$ denotes the variance (channel power) and $\rho_{x y}$ denotes the cross-correlation between the real and the imaginary part. Therefore, for proper data, $\sigma_{x}^{2}=\sigma_{y}^{2}=0$, and $x \perp y$, whereas for improper data, either $\sigma_{x}^{2} \neq \sigma_{y}^{2}$ or the real and imaginary parts are correlated.

In standard strictly linear autoregressive (AR) modeling, the aim is to find a regression of order $p$, given a history of observed data. The standard AR model in $\mathbb{C}$ has the same form as the real valued $A R$ model and is given by

$$
z_{k}=\sum_{i=1}^{p} h_{i} z_{k-i}+n_{k}=\mathbf{h}_{k}^{T} \mathbf{z}_{k}+n_{k}
$$

where $\mathbf{h}_{k}=\left[h_{1}, \ldots, h_{p}\right]^{T}$ is the coefficient vector, $\mathbf{z}_{k}=$ $\left[z_{k-1}, \ldots, z_{k-p}\right]^{T}$ is the regressor vector, and $n_{k}$ is the doubly white Gaussian (zero-mean) driving noise. This model is restricted by the inherent strictly linear regression, and even for noncircular driving noise, it cannot adequately model the generality of complex signals (improper) [1].

On the other hand, the widely linear AR (WLAR) model provides a unified treatment of proper and improper complex signals and is given by [11], [13]

$$
\begin{aligned}
z_{k} & =\sum_{i=1}^{p} h_{i} z_{k-i}+\sum_{i=1}^{p} g_{i} z_{k-i}^{*}+h_{0} n_{k}+g_{0} n_{k}^{*} \\
& =\mathbf{h}_{k}^{T} \mathbf{z}_{k}+\mathbf{g}_{k}^{T} \mathbf{z}_{k}^{*}+\left[h_{0}, g_{0}\right] \mathbf{n}_{k}^{a}
\end{aligned}
$$

where the symbols $\mathbf{h}_{k}$ and $\mathbf{g}_{k}$ denote respectively the standard and conjugate regression coefficients and $\mathbf{w}_{k}^{a}=\left[h_{0}, g_{0}\right]^{T}$ is the "augmented" weight vector. The performance advantage of WLAR over the strictly linear AR model, $\delta \epsilon^{2}$, can be illustrated using the recently introduced approximate uncorrelating transform as [14]

$$
\delta \epsilon^{2} \sim \frac{\lambda_{c}}{\lambda_{c}^{2}-\lambda_{p}^{2}}
$$

where $\lambda_{c}$ denotes the eigenvalues corresponding to the covariance matrix and $\lambda_{p}$ denotes those corresponding to the pseudocovariance matrix. From (10), the widely linear WLAR model outperforms the standard strictly linear AR model for improper signals (for which $\lambda_{p} \neq 0$ ) and has the same performance as the standard AR model for circular signals $\left(\lambda_{p}=0\right)$ [12], as illustrated in Fig. 1 on the modeling of the improper Ikeda signal.

\section{Widely Linear (Augmented) CLMS (ACLMS)}

The LMS algorithm is the most commonly used stochastic gradient adaptive filtering algorithm which adaptively adjusts the filter coefficients in order to minimize the instantaneous squared error (difference between the desired signal and its estimate-the filter output). Following the same principles as in AR versus WLAR modeling, the standard CLMS algorithm 


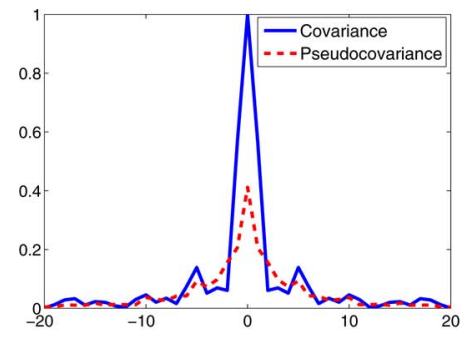

(a)

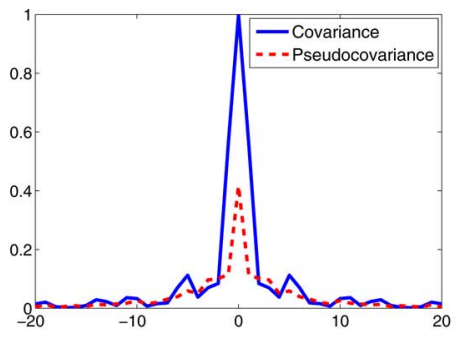

(b)

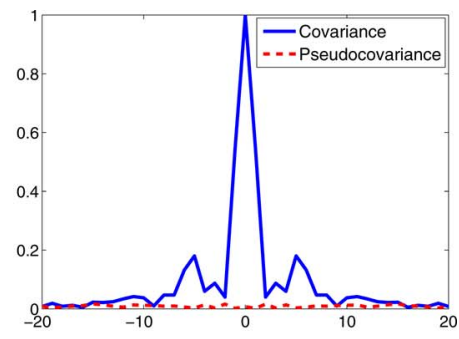

(c)

Fig. 1. Covariance and pseudocovariance of the strictly linear AR(4) and widely linear WLAR(4) models of the improper Ikeda map. (a) Original Ikeda map. (b) WLAR(Ikeda). (c) Standard AR(Ikeda).

is only suited to signals with second-order circular distributions (proper). With this in mind, the widely linear (augmented) CLMS (ACLMS) algorithm has been proposed to cater for both circular and noncircular signals [11] and is summarized in Algorithm 1, where $d_{k}$ is the desired signal at time instant $k$, $\mathbf{h}_{k}$ and $\mathbf{g}_{k}$ are the standard and conjugate filter weights while $\mathbf{w}_{k}^{a}=\left[\mathbf{h}_{k}^{T}, \mathbf{g}_{k}^{T}\right]^{T}$ is the augmented weight vector.

\section{Algorithm 1: ACLMS}

Filter output: $y_{k}=\mathbf{w}_{k}^{a T} \mathbf{x}_{k}^{a}$

Error: $e_{k}=d_{k}-y_{k}$

Weight updates:

$$
\underbrace{\left[\begin{array}{c}
\mathbf{h}_{k+1} \\
\mathbf{g}_{k+1}
\end{array}\right]}_{\equiv \mathbf{w}_{k+1}^{a}}=\underbrace{\left[\begin{array}{c}
\mathbf{h}_{k}+\mu e_{k} \mathbf{x}_{k}^{*} \\
\mathbf{g}_{k}+\mu e_{k} \mathbf{x}_{k}
\end{array}\right]}_{\equiv \mathbf{w}_{k}^{a}+\mu e_{k} \mathbf{x}_{k}^{a *}}
$$

\section{ACKF}

We next introduce the augmented complex Kalman filter (ACKF) for frequency estimation in unbalanced three-phase power systems and illuminate its performance advantage in relation to the conventional complex Kalman filter (CCKF). Consider the linear state space model given by

$$
\begin{aligned}
& \mathbf{x}_{k}=\mathbf{F}_{k-1} \mathbf{x}_{k-1}+\mathbf{u}_{k-1} \\
& \mathbf{y}_{k}=\mathbf{H}_{k} \mathbf{x}_{k}+\mathbf{n}_{k}
\end{aligned}
$$

where $\mathbf{x}_{k}$ is the state to be estimated at time instant $k, \mathbf{y}_{k}$ is the observation (or measurement) of the true state, and the vectors $\mathbf{u}_{k}$ and $\mathbf{n}_{k}$ are the zero-mean state and measurement noises, with covariance matrices $\mathbf{R}_{\mathbf{u}, k}$ and $\mathbf{R}_{\mathbf{n}, k}$ and pseudocovariances $\mathbf{P}_{\mathbf{u}, k}$ and $\mathbf{P}_{\mathbf{n}, k}$, while $\mathbf{F}_{k}$ and $\mathbf{H}_{k}$ are the state transition matrix and observation matrices. The corresponding widely linear state space model is defined as [11], [15]

$$
\begin{aligned}
& \mathbf{x}_{k}=\mathbf{F}_{k-1} \mathbf{x}_{k-1}+\mathbf{A}_{k-1} \mathbf{x}_{k-1}^{*}+\mathbf{u}_{k-1} \\
& \mathbf{y}_{k}=\mathbf{H}_{k} \mathbf{x}_{k}+\mathbf{B}_{k} \mathbf{x}_{k}^{*}+\mathbf{n}_{k}
\end{aligned}
$$

and can be expressed in more compact form using augmented models, such that

$$
\begin{aligned}
\mathbf{x}_{k}^{a} & =\mathbf{F}_{k-1}^{a} \mathbf{x}_{k-1}^{a}+\mathbf{u}_{k-1}^{a} \\
\mathbf{y}_{k}^{a} & =\mathbf{H}_{k}^{a} \mathbf{x}_{k}^{a}+\mathbf{n}_{k}^{a}
\end{aligned}
$$

where the augmented $\mathbf{x}_{k}^{a}=\left[\mathbf{x}_{k}^{T}, \mathbf{x}_{k}^{H}\right]^{T}, \mathbf{y}_{k}^{a}=\left[\mathbf{y}_{k}^{T}, \mathbf{y}_{k}^{H}\right]^{T}$, $\mathbf{F}_{k}^{a}=\left[\begin{array}{ll}\mathbf{F}_{k} & \mathbf{A}_{k} \\ \mathbf{A}_{k}^{*} & \mathbf{F}_{k}^{*}\end{array}\right]$, and $\mathbf{H}_{k}^{a}=\left[\begin{array}{ll}\mathbf{H}_{k} & \mathbf{B}_{k} \\ \mathbf{B}_{k}^{*} & \mathbf{H}_{k}^{*}\end{array}\right]$.

The coefficient matrices, $\mathbf{A}$ and $\mathbf{B}$, determine whether the state and observation equations are strictly linear or widely linear. If $\mathbf{A}=\mathbf{0}$ and $\mathbf{B}=\mathbf{0}$, then the state space is strictly linear; however, the augmented state space representation should still be preferred over the linear state space if the state and observation noises are second order noncircular. Consider the covariance matrices of the augmented state and observation noise vectors, $\mathbf{u}_{k}^{a}=\left[\mathbf{u}_{k}^{T}, \mathbf{u}_{k}^{H}\right]^{T}$ and $\mathbf{n}_{k}^{a}=\left[\mathbf{n}_{k}^{T}, \mathbf{n}_{k}^{H}\right]^{T}$, i.e.,

$$
\begin{aligned}
& \mathbf{R}_{\mathbf{u}, k}^{a}=E\left\{\mathbf{u}_{k}^{a} \mathbf{u}_{k}^{a H}\right\}=\left[\begin{array}{ll}
\mathbf{R}_{\mathbf{u}, k} & \mathbf{P}_{\mathbf{u}, k} \\
\mathbf{P}_{\mathbf{u}, k}^{*} & \mathbf{R}_{\mathbf{u}, k}^{*}
\end{array}\right] \\
& \mathbf{R}_{\mathbf{n}, k}^{a}=E\left\{\mathbf{n}_{k}^{a} \mathbf{n}_{k}^{a H}\right\}=\left[\begin{array}{ll}
\mathbf{R}_{\mathbf{n}, k} & \mathbf{P}_{\mathbf{n}, k} \\
\mathbf{P}_{\mathbf{n}, k}^{*} & \mathbf{R}_{\mathbf{n}, k}^{*}
\end{array}\right] .
\end{aligned}
$$

Notice that the pseudocovariances are naturally incorporated. The widely linear minimum MSE estimate $\widehat{\mathbf{x}}_{k \mid k}^{a}$ of $\mathbf{x}_{k}^{a}$, based on the observations $\left\{\mathbf{y}_{1}^{a}, \mathbf{y}_{2}^{a}, \ldots, \mathbf{y}_{k}^{a}\right\}$, can be computed sequentially using the ACKF, which is summarized in Algorithm 2.

Algorithm 2: ACKF

Initialize with:

$$
\begin{aligned}
\widehat{\mathbf{x}}_{0 \mid 0}^{a} & =E\left\{\mathbf{x}_{0}^{a}\right\} \\
\mathbf{M}_{0 \mid 0}^{a} & =E\left\{\left(\mathbf{x}_{0}^{a}-E\left\{\mathbf{x}_{0}^{a}\right\}\right)\left(\mathbf{x}_{0}^{a}-E\left\{\mathbf{x}_{0}^{a}\right\}\right)^{H}\right\}
\end{aligned}
$$

For $k=1,2, \ldots$

$$
\begin{aligned}
\widehat{\mathbf{x}}_{k \mid k-1}^{a} & =\mathbf{F}_{k-1}^{a} \widehat{\mathbf{x}}_{k-1 \mid k-1}^{a} \\
\mathbf{M}_{k \mid k-1}^{a} & =\mathbf{F}_{k-1}^{a} \mathbf{M}_{k-1 \mid k-1}^{a} \mathbf{F}_{k-1}^{a H}+\mathbf{R}_{\mathbf{u}, k-1}^{a} \\
\mathbf{G}_{k}^{a} & =\mathbf{M}_{k \mid k-1}^{a} \mathbf{H}_{k}^{a H}\left[\mathbf{H}_{k}^{a} \mathbf{M}_{k \mid k-1}^{a} \mathbf{H}_{k}^{a H}+\mathbf{R}_{\mathbf{n}, k}^{a}\right]^{-1} \\
\widehat{\mathbf{x}}_{k \mid k}^{a} & =\widehat{\mathbf{x}}_{k \mid k-1}^{a}+\mathbf{G}_{k}^{a}\left(\mathbf{y}_{k}^{a}-\mathbf{H}_{k}^{a} \widehat{\mathbf{x}}_{k \mid k-1}^{a}\right) \\
\mathbf{M}_{k \mid k}^{a} & =\left(\mathbf{I}-\mathbf{G}_{k}^{a} \mathbf{H}_{k}^{a}\right) \mathbf{M}_{k \mid k-1}^{a}
\end{aligned}
$$

After some tedious algebraic manipulations, the mean square error (MSE) difference between CCKF and the widely linear 
ACKF can be written as [12], [15]

$$
\begin{aligned}
\Delta \mathbf{M}_{k}= & \left(\mathbf{P}_{\mathbf{x z}, k, k}-\mathbf{R}_{\mathbf{x z}, k, k} \mathbf{R}_{\mathbf{z}, k}^{-1} \mathbf{P}_{\mathbf{z}, k}\right) \\
& \times\left(\mathbf{R}_{\mathbf{z}, k}^{*}-\mathbf{P}_{\mathbf{z}, k}^{*} \mathbf{R}_{\mathbf{z}, k}^{-1} \mathbf{P}_{\mathbf{z}, k}\right)^{-1} \\
& \times\left(\mathbf{P}_{\mathbf{x z}, k, k}-\mathbf{R}_{\mathbf{x z}, k, k} \mathbf{R}_{\mathbf{z}, k}^{-1} \mathbf{P}_{\mathbf{z}, k}\right)^{H}
\end{aligned}
$$

where $\mathbf{z}_{k}=\left[\mathbf{y}_{1}^{T}, \ldots, \mathbf{y}_{k}^{T}\right]^{T}$ is the observation sequence with covariance $\mathbf{R}_{\mathbf{z}, k}$ and pseudocovariance $\mathbf{P}_{\mathbf{z}, k}$ while $\mathbf{R}_{\mathbf{x z}, k, k}=$ $E\left\{\left(\mathbf{x}_{k}-E\left\{\mathbf{x}_{k}\right\}\right)\left(\mathbf{z}_{k}-E\left\{\mathbf{z}_{k}\right\}\right)^{H}\right\}$ and $\mathbf{P}_{\mathbf{x z}, k, k}=E\left\{\left(\mathbf{x}_{k}-\right.\right.$ $\left.\left.E\left\{\mathbf{x}_{k}\right\}\right)\left(\mathbf{z}_{k}-E\left\{\mathbf{z}_{k}\right\}\right)^{T}\right\}$ are the cross-correlation and pseudocorrelation between the state and observation sequence.

Remark 1: Owing to the positive definiteness of the ma$\operatorname{trix}\left(\mathbf{R}_{\mathbf{z}, k}^{*}-\mathbf{P}_{\mathbf{z}, k}^{*} \mathbf{R}_{\mathbf{z}, k}^{-1} \mathbf{P}_{\mathbf{z}, k}\right)$, the matrix $\Delta \mathbf{M}_{k}$ is always positive semidefinite, and consequently, $\Delta \mathbf{M}_{k}=\mathbf{0}$ only when $\left(\mathbf{P}_{\mathbf{x z}, k, k}-\mathbf{R}_{\mathbf{x z}, k, k}^{-1} \mathbf{R}_{\mathbf{z}, k} \mathbf{P}_{\mathbf{z}, k}\right)=\mathbf{0}$. Therefore, the widely linear ACKF always has the same or better MSE performance than the strictly linear CCKF.

Remark 2: The CCKF and ACKF yield identical performance, i.e., $\Delta \mathbf{M}_{k}=\mathbf{0}$, when the state and observation noises are circular and the state space equations are both strictly linear; otherwise, the ACKF is superior.

Algorithm 3: State Space 1—Linear (SS1-L)

$$
\text { state equation: } \quad x_{k}=x_{k-1}+u_{k-1}
$$

observation equation: $v_{k}=v_{k-1} x_{k}+n_{k}$

\section{WIDELY LINEAR FREQUENCY ESTIMATION}

The instantaneous three-phase voltages are defined as

$$
\begin{aligned}
& v_{a, k}=V_{a, k} \cos (\omega k T+\phi) \\
& v_{b, k}=V_{b, k} \cos (\omega k T+\phi-2 \pi / 3) \\
& v_{c, k}=V_{c, k} \cos (\omega k T+\phi+2 \pi / 3)
\end{aligned}
$$

where $V_{a, k}, V_{b, k}$, and $V_{c, k}$ are the amplitudes of the three-phase voltages at time instant $k, \omega=2 \pi f$ is the angular frequency with $f$ being the system frequency, $T$ is the sampling interval, and $\phi$ is the phase of the fundamental component. Clarke's transformation, given by

$$
\left[\begin{array}{l}
v_{0, k} \\
v_{\alpha, k} \\
v_{\beta, k}
\end{array}\right]=\sqrt{\frac{2}{3}}\left[\begin{array}{ccc}
\frac{\sqrt{2}}{2} & \frac{\sqrt{2}}{2} & \frac{\sqrt{2}}{2} \\
1 & -\frac{1}{2} & -\frac{1}{2} \\
0 & \frac{\sqrt{3}}{2} & -\frac{\sqrt{3}}{2}
\end{array}\right]\left[\begin{array}{l}
v_{a, k} \\
v_{b, k} \\
v_{c, k}
\end{array}\right]
$$

maps the three-phase voltages onto a new domain where they can be conveniently represented by a scalar complex valued signal. In (27), the zero-sequence $v_{0, k}$ vanishes when the system is balanced, i.e., $V_{a, k}=V_{b, k}=V_{c, k}$, while $v_{\alpha, k}=$ $A_{k} \cos (\omega k T+\phi)$ and $v_{\beta, k}=A_{k} \cos (\omega k T+\phi+(\pi / 2))$ are orthogonal. In practice, the zero-sequence $v_{0, k}$ is not consid-

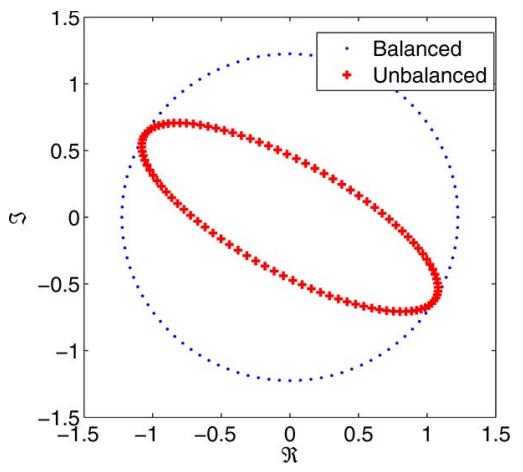

Fig. 2. Geometric view of circularity via a real-imaginary scatter plot of the Clarke voltage $v_{k}$. For a balanced system, characterized by $V_{a, k}=V_{b, k}=$ $V_{c, k}$, the trajectory of $v_{k}$ is circular, while for unbalanced systems, such as in the case of a $100 \%$ single-phase voltage sag illustrated by the ellipse in the figure ("+"), the trajectory of the output voltage becomes noncircular.

ered, and only $v_{\alpha}$ and $v_{\beta}$ are used to form the complex valued model of the system, i.e., ${ }^{2}$

$$
v_{k}=v_{\alpha, k}+j v_{\beta, k}=A_{k} e^{j(\omega k T+\phi)}=v_{k-1} e^{j \omega T} .
$$

The state space model of the system is shown in Algorithm 3, where the state $x_{k}$ is used to estimate the exponential $e^{j \omega T}$ whose argument contains the frequency $f$ and $v_{k}$ is the observation while $u_{k}$ and $n_{k}$ are respectively the state and observation noises. This state space model is linear and can be implemented using the CCKF. The selection of these noise variances is critical to the steady-state error and convergence rates of Kalman filters and will be discussed in more detail in the next section. The system frequency is derived from the state $x$ as

$$
\hat{f}_{k}=\frac{1}{2 \pi T} \arcsin \left(\Im\left(x_{k}\right)\right)
$$

where $\Im(\cdot)$ is the imaginary part of a complex quantity.

Fig. 2 shows that, for a balanced system, Clarke's voltage $v_{k}$ in (28) follows a circular trajectory since the amplitude is time invariant and the angular frequency is proportional to the system frequency. However, this model is inaccurate when the system is operating under unbalanced conditions, in which case the voltage amplitudes $V_{a, k}, V_{b, k}$, and $V_{c, k}$ are no longer equal, and the system trajectory becomes noncircular (ellipse in Fig. 2). Therefore, for unbalanced systems, the true system model becomes widely linear [10], that is,

$$
v_{k}=v_{\alpha, k}+j v_{\beta, k}=A_{k} e^{j(\omega k T+\phi)}+B_{k} e^{-j(\omega k T+\phi)}
$$

with

$$
\begin{aligned}
A_{k} & =\frac{\sqrt{6}\left(V_{a, k}+V_{b, k}+V_{c, k}\right)}{6} \\
B_{k} & =\frac{\sqrt{6}\left(2 V_{a, k}-V_{b, k}-V_{c, k}\right)}{12}-\frac{\sqrt{2}\left(V_{b, k}-V_{c, k}\right)}{4} j .
\end{aligned}
$$

When the system is balanced and operating under nominal conditions, i.e., $V_{a, k}=V_{b, k}=V_{c, k}$, the coefficient $B_{k}$

\footnotetext{
${ }^{2}$ The usual assumption in this type of estimation, that is $A_{k} \approx A_{k-1}$, is used.
} 


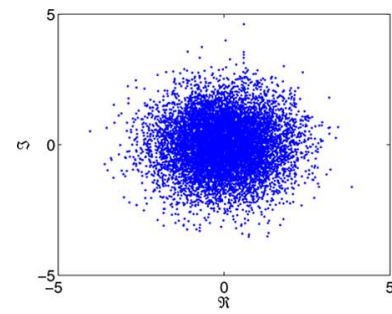

(a) $n_{a}, n_{b}, n_{c} \sim \mathcal{N}(0,1)$

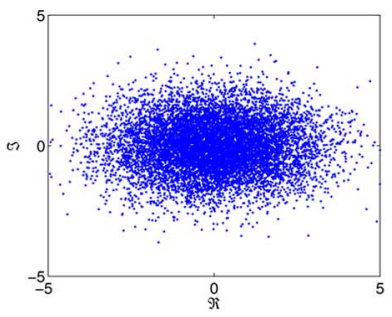

(b) $n_{a} \sim \mathcal{N}(0,3), n_{b}, n_{c} \sim \mathcal{N}(0,1)$

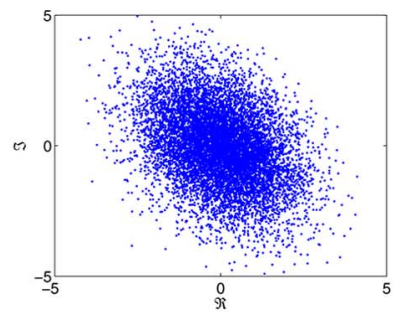

(c) $n_{a}, n_{c} \sim \mathcal{N}(0,1), n_{b} \sim \mathcal{N}(0,3)$

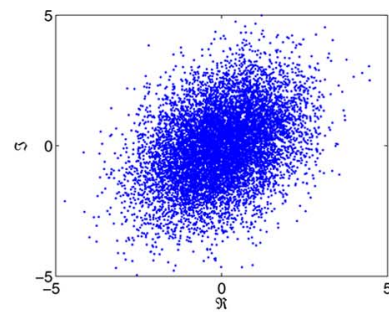

(d) $n_{a}, n_{b} \sim \mathcal{N}(0,1), n_{c} \sim \mathcal{N}(0,3)$

Fig. 3. Observation noise distributions after the three-phase (independent, Gaussian, and real valued) noises $n_{a, k}, n_{b, k}$, and $n_{c, k}$ undergo Clarke's $\alpha \beta$ transformation. (a) $n_{a}, n_{b}, n_{c} \sim \mathcal{N}(0,1)$. (b) $n_{a} \sim \mathcal{N}(0,3), n_{b}, n_{c} \sim \mathcal{N}(0,1)$. (c) $n_{a}, n_{c} \sim \mathcal{N}(0,1), n_{b} \sim \mathcal{N}(0,3)$. (d) $n_{a}, n_{b} \sim \mathcal{N}(0,1), n_{c} \sim \mathcal{N}(0,3)$.

vanishes, and system is accurately characterized by the strictly linear model in (28). Note that the system is noncircular for $B_{k}=0$, the expression in (30) is general and characterizes the system under both balanced and unbalanced conditions, and can be written recursively as

$$
v_{k}=v_{k-1} h_{k-1}+v_{k-1}^{*} g_{k-1}
$$

which is a first-order WLAR model, WLAR(1), addressed in Section II-B. The corresponding widely linear (augmented) state space model is defined in Algorithm 4, where the state vector consists of the strictly linear weight $h_{k}$ and conjugate weight $g_{k}$ and the observation $v_{k}$ is a widely linear function of the previous observation while $\mathbf{u}_{h, k}$ and $\mathbf{u}_{g, k}$ are the state noises corresponding to $h_{k}$ and $g_{k}$.

Algorithm 4: State Space 2-Widely Linear (SS2-WL) state equation:

$$
\left[\begin{array}{c}
h_{k} \\
g_{k} \\
h_{k}^{*} \\
g_{k}^{*}
\end{array}\right]=\left[\begin{array}{c}
h_{k-1} \\
g_{k-1} \\
h_{k-1}^{*} \\
g_{k-1}^{*}
\end{array}\right]+\left[\begin{array}{l}
u_{h, k-1} \\
u_{g, k-1} \\
u_{h, k-1}^{*} \\
u_{g, k-1}^{*}
\end{array}\right]
$$

observation equation:

$$
v_{k}=\left[\begin{array}{cccc}
v_{k-1} & v_{k-1}^{*} & 0 & 0 \\
0 & 0 & v_{k-1}^{*} & v_{k-1}
\end{array}\right]\left[\begin{array}{c}
h_{k} \\
g_{k} \\
h_{k}^{*} \\
g_{k}^{*}
\end{array}\right]+\left[\begin{array}{l}
n_{k} \\
n_{k}^{*}
\end{array}\right]
$$

The system frequency is computed from

$$
\begin{aligned}
\hat{f}_{k} & =\frac{1}{2 \pi T} \arcsin \left(\Im\left(h_{k}+a_{k} g_{k}\right)\right) \\
a_{k} & =\frac{-j \Im\left(h_{k}\right)+j \sqrt{\Im^{2}\left(h_{k}\right)-\left|g_{k}\right|^{2}}}{g_{k}} .
\end{aligned}
$$

Central to both the state space models SS1 and SS2 is the assumption of noise-free observations. In fact, if the threephase voltages $v_{a, k}, v_{b, k}$, and $v_{c, k}$ are corrupted by additive real valued zero-mean noises $n_{a, k}, n_{b, k}$, and $n_{c, k}$, respectively, then the output voltage is given by

$$
v_{k}=v_{\alpha, k}+j v_{\beta, k}+n_{k} .
$$

From Clarke's transform, the $\alpha \beta$ transformed noise is

$$
\begin{aligned}
n_{k}= & n_{\alpha, k}+j n_{\beta, k} \\
= & \sqrt{2 / 3}\left(n_{a, k}-\frac{1}{2} n_{b, k}-\frac{1}{2} n_{c, k}\right) \\
& +j \sqrt{2 / 3}\left(\frac{\sqrt{3}}{2} n_{b, k}-\frac{\sqrt{3}}{2} n_{c, k}\right)
\end{aligned}
$$

which is a zero-mean complex noise with pseudocovariance

$$
\begin{aligned}
p_{n, k}= & E\left\{n_{k}^{2}\right\} \\
=E & \left\{\frac{2}{3} n_{a, k}^{2}-\frac{1}{3} n_{b, k}^{2}-\frac{1}{3} n_{c, k}^{2}-\frac{2}{3} n_{a, k} n_{b, k}\right. \\
& \left.\quad-\frac{2}{3} n_{a, k} n_{c, k}--\frac{4}{3} n_{b, k} n_{c, k}\right\} \\
\times j E & \left\{\frac{2 \sqrt{3}}{3} n_{a, k} n_{b, k}-\frac{2 \sqrt{3}}{3} n_{a, k} n_{c, k}\right. \\
& \left.\quad-\frac{2 \sqrt{3}}{3} n_{b, k}^{2}+\frac{2 \sqrt{3}}{3} n_{c, k}^{2}\right\} .
\end{aligned}
$$

The circularity of the noise $n_{k}$ is determined by the ratios of the variances and cross-correlations of the three-phase observation noises $n_{a, k}, n_{b, k}$, and $n_{c, k}$. Fig. 3 shows that the transformed noise $n_{k}$ is proper, if and only if, the three-phase noises are all uncorrelated and have identical variances; otherwise, it is improper. Thus, equal line noise powers provide a circular Clarke's noise, whereas combinations of different noise powers provide noncircular Clarke's noises with different degrees and natures of improperness. For instance, the case in Fig. 3(b) is improper with $n_{\alpha, k} \perp n_{\beta, k}$, whereas the cases in Fig. 3(c) and (d) are improper with $n_{\alpha, k}$ and $n_{\beta, k}$ exhibiting different characters of correlations. Therefore, the noncircularity of noise should be dealt with within the algorithm structure.

In the presence of noise, the recursion for Clarke's voltage can be found by substituting (36) into (32), i.e.,

$$
\begin{aligned}
v_{k} & =\left(v_{k-1}+n_{k-1}\right) h_{k-1}+\left(v_{k-1}^{*}+n_{k-1}^{*}\right) g_{k-1}+n_{k} \\
& =v_{k-1} h_{k-1}+v_{k-1}^{*} g_{k-1}+n_{k-1} h_{k-1}+n_{k-1}^{*} g_{k-1}+n_{k} .
\end{aligned}
$$


Remark 3: In (39), the observation noise $n_{k}$ in (39) is additive, while the previous noise $n_{k-1}$ has a multiplicative effect on the current observation. However, in practice, the dynamics of real-world three-phase systems do not follow this model, i.e., the current observation is not a function of the previous observation noise, and using (39) to model noisy real-world systems can lead to degraded or diverging estimates.

We next propose a more realistic sequential state space model where the current observation is independent of the previous observation noises. This is achieved by also including a widely linear estimate of the output voltage within the state vector, as summarized in Algorithm 5.

Algorithm 5: State Space 3-Widely Linear (SS3-WL) state equation:

$$
\left[\begin{array}{c}
h_{k} \\
g_{k} \\
\hat{v}_{k} \\
h_{k}^{*} \\
g_{k}^{*} \\
\hat{v}_{k}^{*}
\end{array}\right]=\left[\begin{array}{c}
h_{k-1} \\
g_{k-1} \\
\hat{v}_{k-1} h_{k-1}+\hat{v}_{k-1}^{*} g_{k-1} \\
h_{k-1}^{*} \\
g_{k-1}^{*} \hat{v}_{k-1}^{*} h_{k-1}^{*}+\hat{v}_{k-1} g_{k-1}^{*}
\end{array}\right]+\mathbf{u}_{k-1}
$$

observation equation:

$$
\left[\begin{array}{c}
v_{k} \\
v_{k}^{*}
\end{array}\right]=\left[\begin{array}{llllll}
0 & 0 & 1 & 0 & 0 & 0 \\
0 & 0 & 0 & 0 & 0 & 1
\end{array}\right]\left[\begin{array}{c}
h_{k} \\
g_{k} \\
\hat{v}_{k} \\
h_{k}^{*} \\
g_{k}^{*} \\
\hat{v}_{k}^{*}
\end{array}\right]+\left[\begin{array}{c}
n_{k} \\
n_{k}^{*}
\end{array}\right]
$$

Remark 4: The state space model in Algorithm 5 does not use the previous observation to form the current observation and hence does not propagate previous observation noises, thus providing a more a realistic and robust characterization of realworld power systems. The state equation is nonlinear due to the coupling between $v$ and $x$ and can be implemented using the augmented complex extended and unscented Kalman filters, ACEKF and ACUKF [15].

A strictly linear version of the state space model defined by (40) and (41) was proposed in [3], where the output voltage was estimated using the strictly linear model in (28). This state space model is described in Algorithm 6.

Algorithm 6: State Space 4-Linear (SS4-L)

state equation:

$$
\left[\begin{array}{c}
x_{k} \\
\hat{v}_{k}
\end{array}\right]=\left[\begin{array}{c}
x_{k-1} \\
\hat{v}_{k-1} x_{k}
\end{array}\right]+\mathbf{u}_{k-1}
$$

observation equation:

$$
v_{k}=\left[\begin{array}{ll}
0 & 1
\end{array}\right]\left[\begin{array}{l}
x_{k} \\
\hat{v}_{k}
\end{array}\right]+n_{k}
$$

Remark 5: Owing to its strictly linear nature, the existing model in Algorithm 6 suffers from the same limitations as the state space model described by Algorithm 3, namely, it is not suited to systems operating in unbalanced conditions and in the presence of voltage sags or transients.

\section{Robust Tracking Using the InNovation Process}

The covariances of the state and observation noises govern the steady-state error as well as the convergence speed of Kalman filters [16]. For example, when estimating constants, for a fixed observation noise variance, the smaller we set the state noise variance, the slower the convergence rate will be. The noise statistics should ideally be matched to the system operating conditions: Harmonic and random interferences should be reflected in the statistics of the observation noise, while changes in the system dynamics, such as voltage sags, should be reflected in the state process.

Solutions for the estimation of the statistics of the state and observations noises mostly assume a degree of stationarity. However, in a real-world power system, the true noise statistics are generally unknown and almost invariably nonstationary, and the exact time instances at which changes occur in the system are generally unpredictable.

To cater for these uncertainties, we propose to employ the innovation process $\nu_{k}=\mathbf{y}_{k}^{a}-\mathbf{H}_{k}^{a} \widehat{\mathbf{x}}_{k \mid k-1}^{a}$ within the Kalman filter, i.e., the difference between the actual and predicted observations, and use large changes in the innovation as an indication of changes in the system dynamics. To this end, we first show that, if the state and observation noise covariance matrices are simultaneously scaled by the same factor, the Kalman gain and state estimate are unaltered [17]. In other words, it is the ratio between the state and observation noise variances which determines the Kalman gain and not the exact values of these variances; hence, the actual noise variances need not be known. Mathematically, the Kalman gain $\mathbf{G}_{k}^{a}$ can be written in a recursive form, where the time updates for the predicted state covariance matrix $\mathbf{M}_{k \mid k-1}^{a}$ can be expressed as

$$
\begin{aligned}
\mathbf{M}_{k+1 \mid k}^{a}= & \mathbf{F}_{k-1}^{a} \mathbf{M}_{k \mid k-1}^{a} \mathbf{F}_{k-1}^{a H}-\mathbf{F}_{k-1}^{a} \mathbf{M}_{k \mid k-1}^{a} \mathbf{H}_{k}^{a H} \\
& \times\left[\mathbf{H}_{k}^{a} \mathbf{M}_{k \mid k-1}^{a} \mathbf{H}_{k}^{a H}+\mathbf{R}_{\mathbf{n}, k}^{a}\right]^{-1} \\
& \times \mathbf{H}_{k}^{a} \mathbf{M}_{k \mid k-1}^{a} \mathbf{F}_{k-1}^{a H}+\mathbf{R}_{\mathbf{u}, k-1}^{a}
\end{aligned}
$$

i.e., via a Riccati recursion, with initial condition $\mathbf{M}_{0 \mid-1}^{a}=\mathbf{M}_{0}^{a}$. The computations for $\mathbf{M}_{k \mid k-1}^{a}$ and $\mathbf{M}_{k \mid k}^{a}$ are independent of the observations and can be calculated without any knowledge of the observations. The state covariance matrix $\mathbf{M}_{k \mid k}^{a}$ can be computed from $\mathbf{M}_{k \mid k-1}^{a}$ by substituting expression (20) into (22) and using the matrix inversion lemma, i.e.,

$$
\begin{aligned}
\mathbf{M}_{k \mid k}^{a}= & \mathbf{M}_{k \mid k-1}^{a}-\mathbf{M}_{k \mid k-1}^{a} \mathbf{H}_{k}^{a H} \\
& \times\left[\mathbf{H}_{k}^{a} \mathbf{M}_{k \mid k-1}^{a} \mathbf{H}_{k}^{a H}+\mathbf{R}_{\mathbf{n}, k}^{a}\right]^{-1} \mathbf{H}_{k}^{a} \mathbf{M}_{k \mid k-1}^{a} \\
= & {\left[\left(\mathbf{M}_{k \mid k-1}^{a}\right)^{-1}+\mathbf{H}_{k}^{a H}\left(\mathbf{R}_{\mathbf{n}, k}^{a}\right)^{-1} \mathbf{H}_{n}^{a}\right]^{-1} }
\end{aligned}
$$


Substitute (45) into (20) to give the Kalman gain

$$
\begin{aligned}
\mathbf{G}_{k} & =\left[\left(\mathbf{M}_{k \mid k-1}^{a}\right)^{-1}+\mathbf{H}_{k}^{a H}\left(\mathbf{R}_{\mathbf{n}, k}^{a}\right)^{-1} \mathbf{H}_{n}^{a}\right]^{-1} \mathbf{H}_{k}^{a H}\left(\mathbf{R}_{\mathbf{n}, k}^{a}\right)^{-1} \\
& =\mathbf{M}_{k \mid k}^{a} \mathbf{H}_{k}^{a H}\left(\mathbf{R}_{\mathbf{n}, k}^{a}\right)^{-1} .
\end{aligned}
$$

Rearrange (46) so that

$$
\mathbf{M}_{k \mid k}^{a}=\mathbf{G}_{k} \mathbf{R}_{\mathbf{n}, k}^{a}\left(\mathbf{H}_{k}^{a H}\right)^{-1}
$$

and substitute into (20) to obtain a recursive expression for the Kalman gain

$$
\begin{aligned}
\mathbf{G}_{k}=[ & {\left[\mathbf{F}_{k-1}^{a} \mathbf{G}_{k-1} \mathbf{R}_{\mathbf{n}, k}^{a}\left(\mathbf{H}_{k}^{a H}\right)^{-1} \mathbf{F}_{k-1}^{a H}+\mathbf{R}_{\mathbf{u}, k-1}^{a}\right] \mathbf{H}_{k}^{a H} } \\
& \times\left[\mathbf{H}_{k}^{a} \mathbf{F}_{k-1}^{a} \mathbf{G}_{k-1} \mathbf{R}_{\mathbf{n}, k}^{a}\left(\mathbf{H}_{k}^{a H}\right)^{-1} \mathbf{F}_{k-1}^{a H} \mathbf{H}_{k}^{a H}\right. \\
& \left.\quad+\mathbf{H}_{k}^{a} \mathbf{R}_{\mathbf{u}, k-1}^{a} \mathbf{H}_{k}^{a H}+\mathbf{R}_{\mathbf{n}, k}^{a}\right]^{-1}
\end{aligned}
$$

Remark 6: The recursion in (48) shows that the Kalman gain is unchanged if the covariance matrices of state and observation noises are both scaled by the same factor, i.e., using $\kappa \mathbf{R}_{\mathbf{u}, k-1}^{a}$ and $\kappa \mathbf{R}_{\mathbf{n}, k}^{a}$ yields the same Kalman gain and state estimate as when using the true noise covariances $\mathbf{R}_{\mathbf{u}, k-1}^{a}$ and $\mathbf{R}_{\mathbf{n}, k}^{a}$, where $\kappa>0$.

Based on (48), the observation noise variance can be set to a positive constant, while the state noise variance can be chosen to balance between the convergence speed and the steady-state error. With this in mind, for the remainder of this section, the observation noise variance is assumed fixed.

Imposing a low state noise variance would lead to a low steady-state error; however, the Kalman filter will be unable to quickly track sudden changes in the system and vice versa for relatively high state noise variance. If significant changes in system dynamics are to be detected, then a large state noise variance should be set when these changes occur while maintaining a low state noise variance if otherwise. ${ }^{3}$

With this setup, we can preserve fast convergence together with low steady-state error. A convenient way of detecting changes in system dynamics is to monitor the innovation $\nu_{k}$. Large changes in the innovation indicate that the observed signal does not match the Kalman filter state estimate, and consequently, the state estimate is inaccurate. We propose to mitigate this issue by setting the state noise variance (or state covariance matrix $\kappa \mathbf{M}_{k \mid k}^{a}$ ) to a relatively large value so that the state is reestimated from the observations.

A simple estimate of the innovation power is an $L$ sample moving average ${ }^{4}$ given by

$$
\left|\bar{\nu}_{k}\right|^{2}=\frac{1}{L} \sum_{i=k-L-1}^{k}\left|\nu_{i}\right|^{2} .
$$

At time $k$, if $\left|\nu_{k}\right|^{2}>c\left|\bar{\nu}_{k-1}\right|^{2}$, where $c>1$ is a threshold, then the state estimate is considered inaccurate, and the state noise

\footnotetext{
${ }^{3}$ The same effect can be achieved by setting a low state noise variance and setting the state covariance $\mathbf{M}_{k \mid k}^{a}$ as $\kappa \mathbf{M}_{k \mid k}^{a}$ with $\kappa \gg 1$ when a system change is detected.

${ }^{4}$ In power systems operating at $50 \mathrm{~Hz}$, the cycle period is $20 \mathrm{~ms}$, and it is of interest to estimate changes in the system frequency in less than the duration of this cycle; hence, the corresponding number of samples, $L$, has to also be smaller than the cycle period.
}
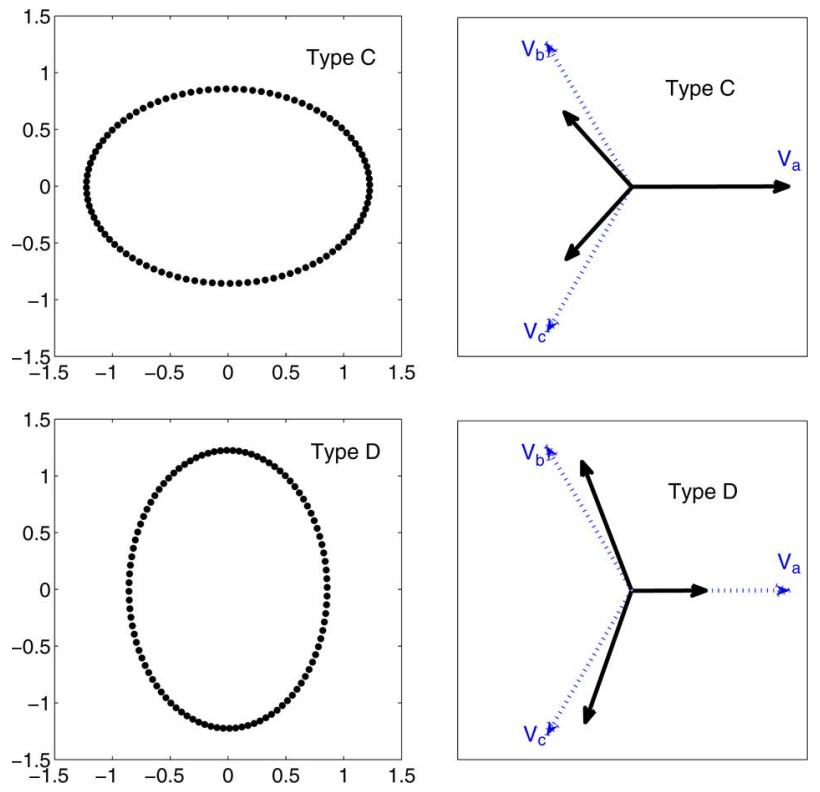

Fig. 4. Geometric and phasor views of Type $\mathrm{C}$ and Type D voltage sags. The real-imaginary plots illustrate the noncircularity of Clarke's voltage in unbalanced conditions. The parameters of the circularity plot (ellipse) help identify the type of fault (voltage sag).

variance is increased for the next time instant. This allows for the detection of changes in system dynamics (e.g., the occurrence of voltage sags), and hence, the noise variances can be set accordingly.

\section{Simulations}

The proposed widely linear sequential state estimation algorithms were assessed for a simulated benchmark system using a $5-\mathrm{kHz}$ sampling rate and were all initialized to $50.5 \mathrm{~Hz}$. The strictly linear state space models, SS1 and SS2, were implemented using the CCKF, and SS4 was implemented using the conventional complex extended Kalman filter (CEKF), while the (widely linear) augmented CEKF (ACEKF) was used for SS3 [15]. Their performances were compared with those of their stochastic gradient-based counterparts, the strictly linear CLMS and widely linear ACLMS [11].

In the first set of simulations, the performances of the algorithms were evaluated for an initially balanced system which became unbalanced after undergoing a Type $C$ voltage sag starting at $0.1 \mathrm{~s}$, characterized by a $20 \%$ voltage drop and a $10^{\circ}$ phase offset on both $v_{b}$ and $v_{c}$, followed by a Type $\mathrm{D}$ sag starting at $0.25 \mathrm{~s}$, characterized by a $20 \%$ voltage drop at line $v_{a}$ and a $10 \%$ voltage drop on both $v_{b}$ and $v_{c}$ with a $5^{\circ}$ phase angle offset, as illustrated in Fig. 4. Observe from Fig. 5 that, for an unbalanced system, the widely linear algorithms, ACLMS, SS2, and SS3, were able to accurately estimate the system frequency, conforming with the analysis, while the strictly linear algorithms, CLMS, SS1, and SS4, yielded oscillating frequency estimates due to the undermodeling of the system. The widely linear and strictly linear algorithms had similar performances under balanced conditions, as illustrated in the time interval $0-0.1 \mathrm{~s}$. Due to their stochastic gradient nature, CLMS and ACLMS had relatively slow convergence compared with the state space-based Kalman filter algorithms, as illustrated in Fig. 6. 

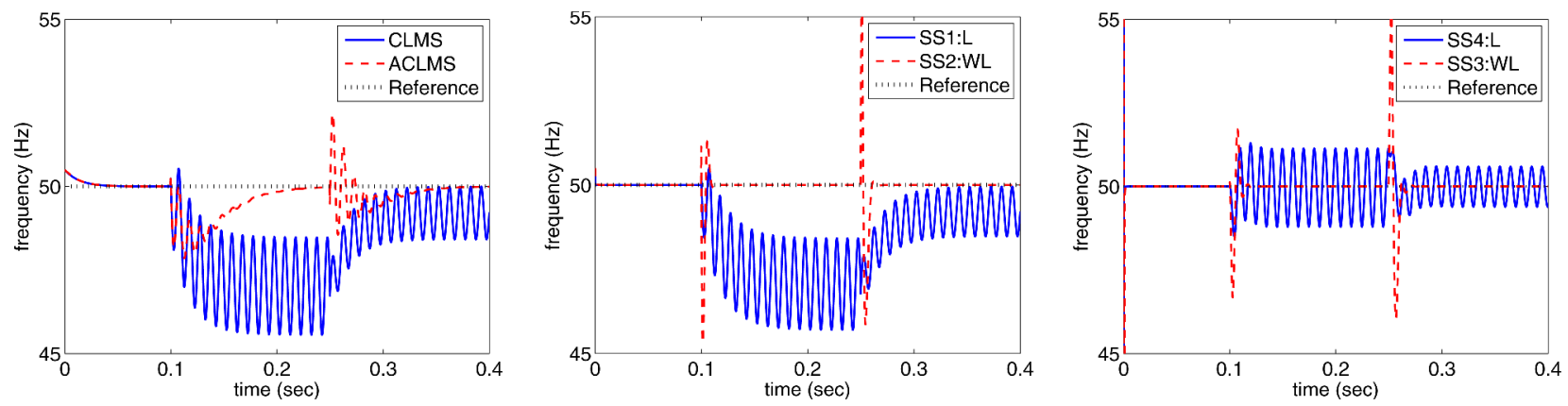

Fig. 5. Frequency estimation for a system which is balanced up to $0.1 \mathrm{~s}$, after which the system becomes unbalanced due to the occurrence of voltage sags of differing natures.
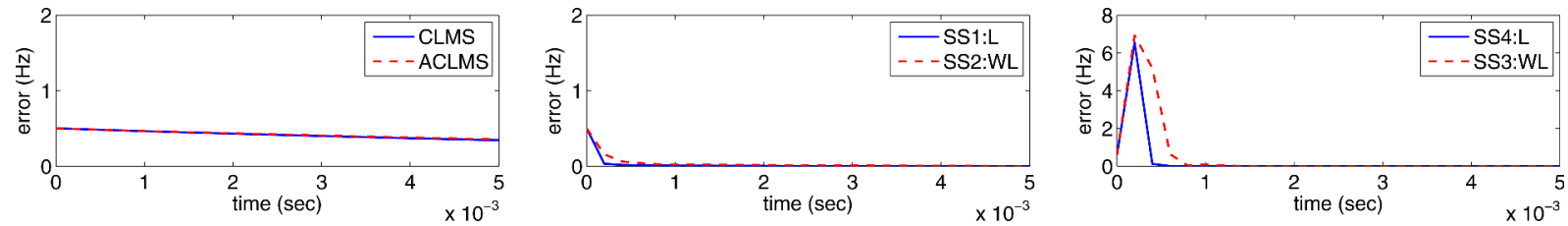

Fig. 6. Initial transient behavior for the simulations in Fig. 5 (first $5 \mathrm{~ms}$ ), where all the Kalman filters where initialized as $\mathbf{M}_{k \mid k}^{a}=10 \mathbf{I}$.
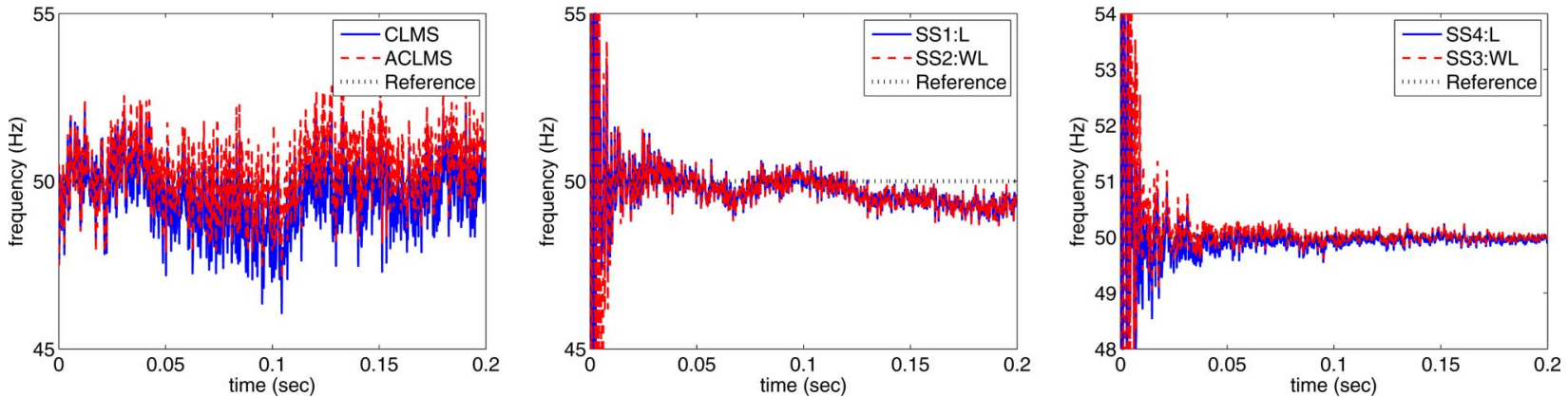

Fig. 7. Frequency estimation for a balanced system in the presence of doubly white circular Gaussian noises at 20-dB SNR.
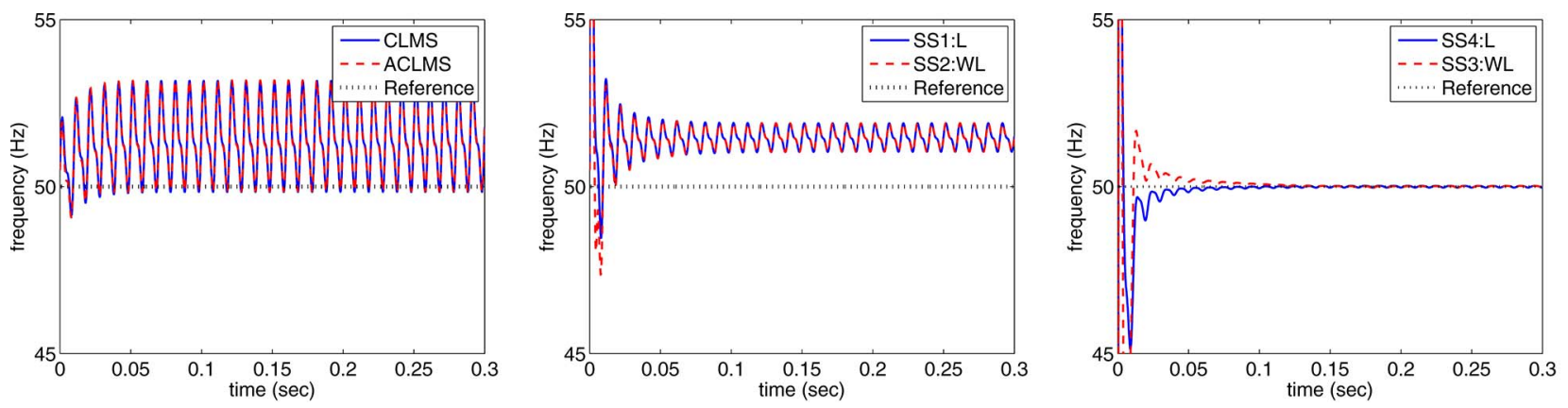

Fig. 8. Frequency estimation when phase voltages are contaminated with in-phase harmonics at $10 \%$ p.u. for the 3 rd and $5 \%$ p.u. for the 5 th harmonics.

Fig. 7 illustrates the frequency estimation in the presence of Gaussian noise. As expected, CLMS, ACLMS, SS1, and SS2, which assume noise-free observations, gave inaccurate estimates, while the more general SS3 and SS4 provided accurate frequency estimates. Fig. 8 illustrates the frequency estimation in the presence of in-phase harmonic observation noise. Again, only SS3 and SS4, which do not assume noisefree observations, converged to the true system frequency, and the remaining algorithms gave inaccurate estimates.
The performance of the algorithms for a power system which undergoes rise and decay in frequency, a typical case where generation does not match the load like in microgrids and islanding, is illustrated in Fig. 9. The widely linear algorithms, ACLMS, SS2, and SS3, were able to accurately track the system frequency, as opposed to their corresponding strictly linear counterparts. Moreover, the stochastic gradient-based ACLMS was outperformed by the widely linear Kalman filter algorithms, SS2 and SS3. 

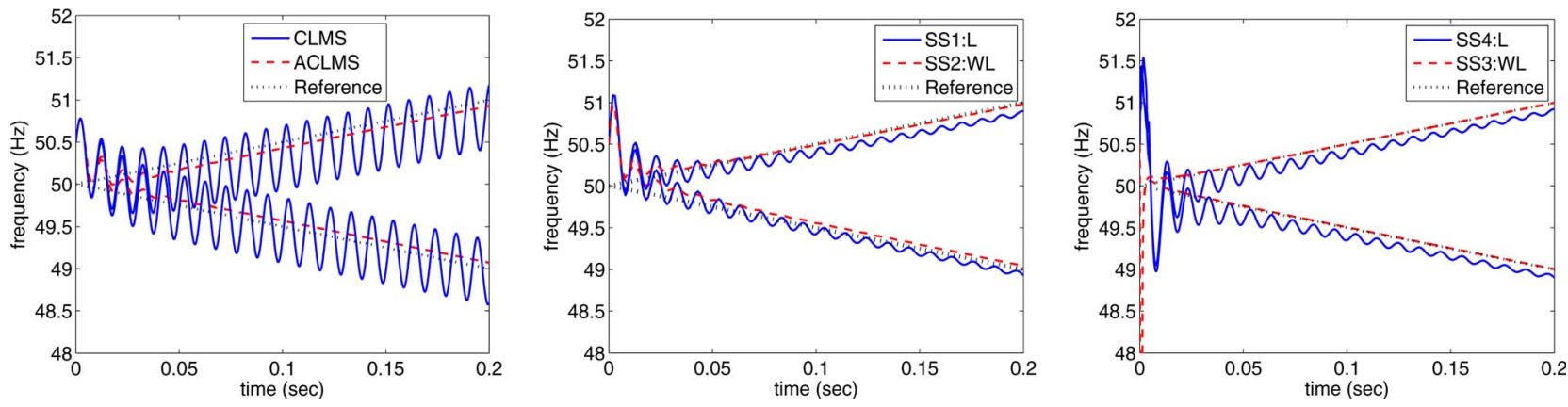

Fig. 9. Frequency estimation for a power system which experiences a 5-Hz/s rise and decay in system frequency.

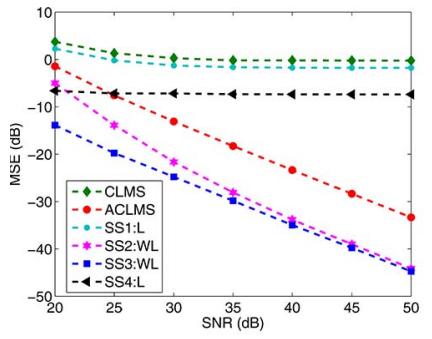

(a)

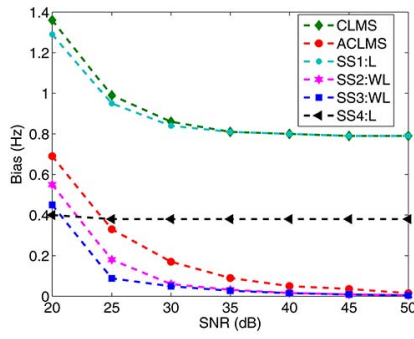

(b)

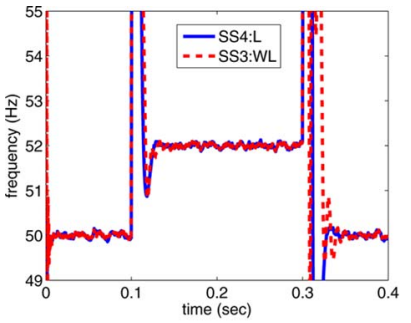

(a) Fixed noise variances

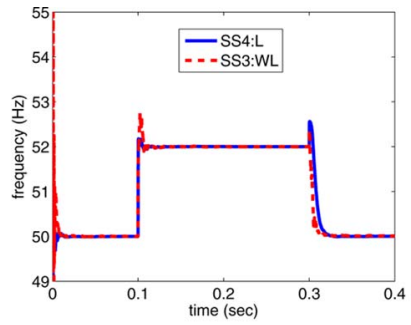

(b) Noise variances set according to in novation power

Fig. 10. MSE and bias analysis for an unbalanced system undergoing a voltage sag (Type D). (a) MSE analysis. (b) Bias analysis.

The statistical advantage of the widely linear estimators over their corresponding strictly linear estimators is illustrated by comparing the bias and MSEs in the presence of Gaussian doubly white circular complex noise. ${ }^{5}$ Fig. 10 shows the performance of the algorithms for a system undergoing a Type D voltage sag (see Fig. 4). The results in Fig. 10(a) illustrate that the widely linear algorithms, ACLMS, SS2, and SS3, had decreasing MSEs as the signal-to-noise ratio (SNR) increased, while the strictly linear algorithms, CLMS, SS1, and SS4, yielded relatively large, almost constant, MSEs with increasing SNR. This can be attributed to the oscillating frequency estimates of these algorithms for unbalanced conditions that do not change with increasing SNR. Moreover, SS3 and SS4 had the best performances among the widely linear and strictly linear algorithms, respectively, because they did not assume noisefree observations. Fig. 10(b) shows the bias of the algorithms at different SNRs; observe that the algorithms based on the widely linear model offered the best performances and that, again, the best results among the strictly linear and widely linear algorithms were achieved by SS4 and SS3, respectively. In other words, Fig. 10 shows that the widely linear algorithms were asymptotically unbiased and statistically consistent.

Fig. 11 illustrates the benefits of monitoring the innovation process to adjust the model to the changes in the system, evaluated for a system which undergoes a step change in frequency in the presence of additive white Gaussian observation noise using SS3 and SS4 (similar results can be shown for the other

\footnotetext{
${ }^{5}$ For white Gaussian noise, $n_{k}=n_{r, k}+j n_{i, k}$, double whiteness implies $E\left\{n_{k} \cdot n_{l}^{*}\right\}=\sigma^{2} \delta_{k-l}$ and $E\left\{n_{k} \cdot n_{l}\right\}=\rho^{2} \delta_{k-l}$, where $\sigma^{2}$ and $\rho^{2}$ are the noise variance and pseudovariance.
}

Fig. 11. Frequency estimation for a system which experiences a temporary step change in frequency from 50 to $52 \mathrm{~Hz}$ in the presence of doubly white circular Gaussian noises at 35-dB SNR. In (a), the frequency is estimated using SS3 and SS4 with fixed state and observation noise variances, while in (b), the state noise variance was set according to the innovation power using the methodology described in Section V. (a) Fixed noise variances. (b) Noise variances set according to innovation power.

state space models). Observe the superior frequency estimation results, in terms of convergence speed and steady-state error, when the state noise variance was set according to the changes in the innovation process, compared to a conventional Kalman filter implementation.

The robustness of the proposed models to a combination of harmful events was examined using the setup in Fig. 5, where an initially balanced system experienced consecutive voltage sags, together with the presence of doubly white Gaussian noise at 40-dB SNR, with the results shown in Fig. 12. Observe that, when the noise variances were set according to innovation power, the algorithm was more robust in the presence of the noise. Fig. 12(c) shows the two peaks in the innovation process corresponding to the time instances when the system experienced the two different voltage sags.

The last set of simulations considers frequency estimation for a real-world power system, where unbalanced three-phase voltages were recorded at a $110 / 20 / 10 \mathrm{kV}$ transformer station. The measured three "phase-ground" voltages come from a system with a nominal frequency of $50 \mathrm{~Hz}$, sampled at a rate of $1 \mathrm{kHz}$ and normalized with respect to their normal peak voltage value. The first set of results, for an unbalanced system (a single-phase short with earth), is shown in Fig. 13, where the theoretical and practical superiority of the algorithms based on the widely linear model, ACLMS and SS3, compared with the strictly linear algorithms, CLMS and SS4, in unbalanced 


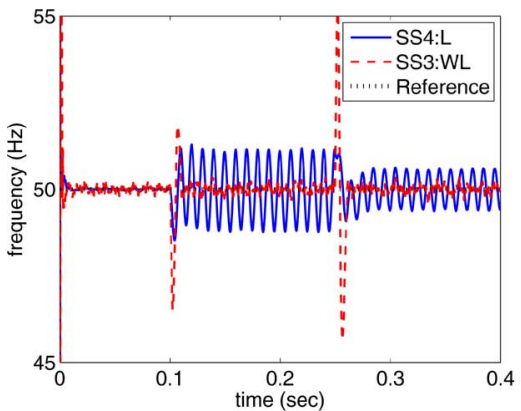

(a)

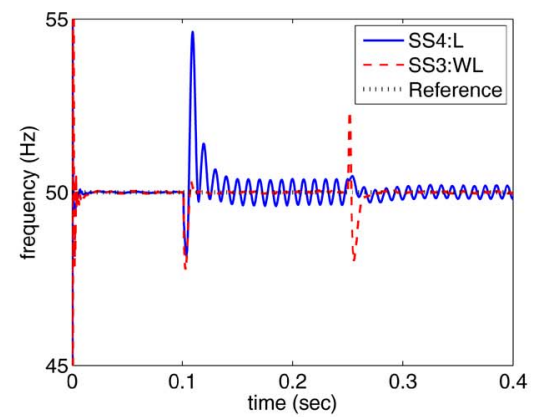

(b)

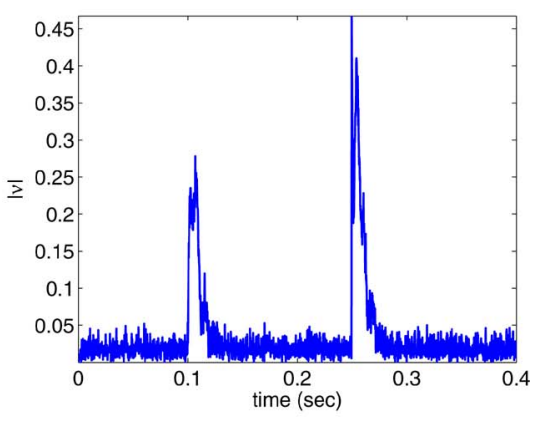

(c)

Fig. 12. An initially balanced system experiences a series of voltages sags, all in the presence of complex doubly white measurement noise. (a) Fixed noise variances. (b) Noise variances set according to innovation power. (c) Innovation signal for SS3.
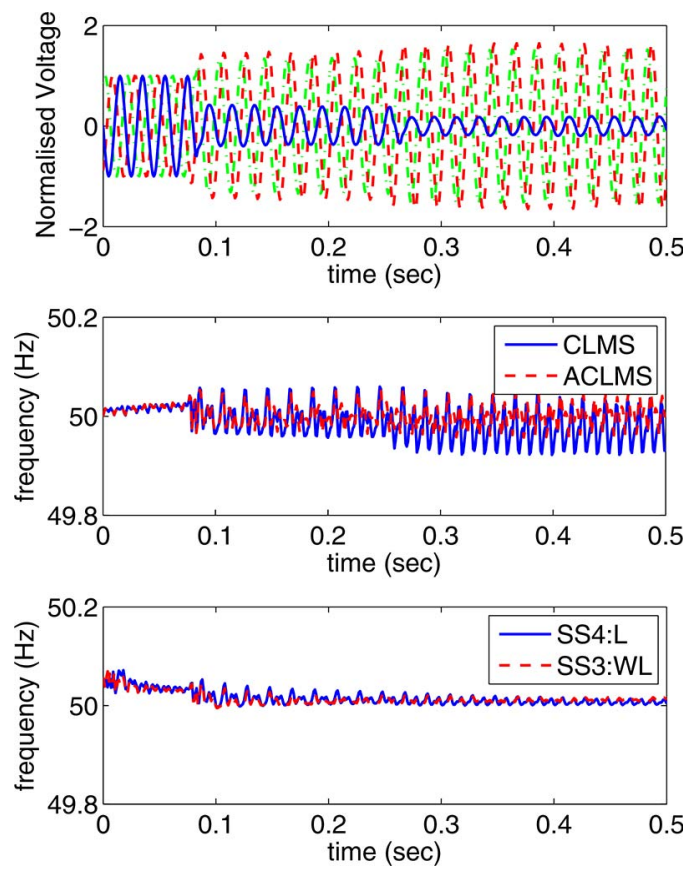

Fig. 13. Frequency estimation for a real-world three-phase system, where an initially balanced system experienced a single-line short with earth.

system conditions is highlighted. Fig. 14 illustrates the performance of the algorithms for a real-world unbalanced system undergoing a two-phase shortcut with earth. Conforming with the analysis, the strictly linear algorithms, CLMS and SS4, yielded inaccurate, biased, and oscillating frequency estimates due to the undermodeling of the system, while the algorithms based on the widely linear model, ACLMS and SS3, yielded accurate estimates, which were unbiased and with minimum variance, conforming with the ensemble analysis in Fig. 10. In both simulations, the state space-based widely linear Kalman filterbased algorithm, SS3, had a faster convergence rate and lower steady-state error than the stochastic gradient-based widely linear ACLMS algorithm.

\section{CONCLUSION}

We have introduced a novel widely linear framework for state space-based frequency estimation in the context of threephase power systems, under both balanced and unbalanced
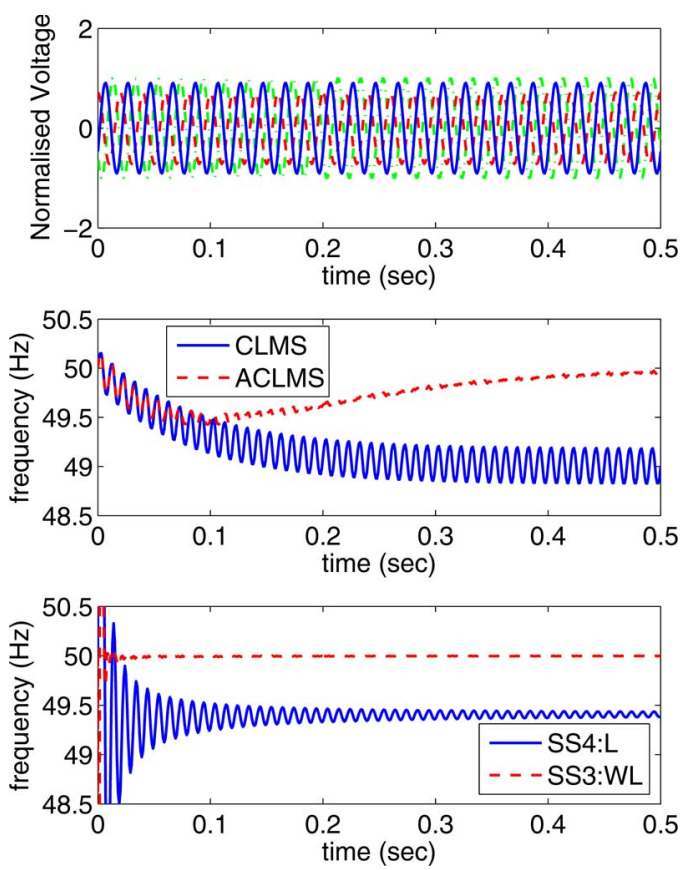

Fig. 14. Frequency estimation for a real-world unbalanced three-phase system, where two lines experience a short with earth.

operating conditions. The signal, obtained from Clarke's $\alpha \beta$ transformation, is noncircular (improper) when the three-phase voltages are unbalanced, which makes the standard strictly linear estimation inadequate. It has been shown that accounting for noncircularity of amplitude distributions allows for both the development of second-order optimal frequency estimation algorithms and the identification of unbalanced conditions via circularity diagrams and degrees of impropriety. We have addressed frequency estimation from a state space perspective and illustrated the superiority of the widely linear (augmented) complex Kalman filters over the stochastic gradient-based ACLMS algorithm. In order to increase the convergence speed and reduce the steady-state error, a method based on the Kalman filter innovation process has also been proposed and was shown to enhance the performance of the Kalman filters in terms of response and convergence rate. Comprehensive simulations over a range of power system conditions evaluated for both balanced and unbalanced systems support the analysis. 


\section{ACKNOWLEDGMENT}

This work is part of the University Defense Research Centre at Imperial College London, supported by the Ministry of Defense (MoD) and Defense Science \& Technology Laboratory (DSTL).

\section{REFERENCES}

[1] Y. Xiao, R. K. Ward, L. Ma, and A. Ikuta, "A new LMS-based Fourier analyzer in the presence of frequency mismatch and applications," IEEE Trans. Circuits Syst. I, Reg. Papers, vol. 52, no. 1, pp. 230-245, Jan. 2005.

[2] C. H. Huang, C. H. Lee, K. J. Shih, and Y. J. Wang, "Frequency estimation of distorted power system signals using a robust algorithm," IEEE Trans. Power Del., vol. 23, no. 1, pp. 41-51, Jan. 2008.

[3] P. K. Dash, A. K. Pradhan, and G. Panda, "Frequency estimation of distorted power system signals using extended complex Kalman filter," IEEE Trans. Power Del., vol. 14, no. 3, pp. 761-766, Jul. 1999.

[4] M. Wang and Y. Sun, "A practical, precise method for frequency tracking and phasor estimation," IEEE Trans. Power Del., vol. 19, no. 4, pp. 15471552, Oct. 2004

[5] T. Lobos and J. Rezmer, "Real-time determination of power system frequency," IEEE Trans. Instrum. Meas., vol. 46, no. 4, pp. 877-881, Aug. 1997.

[6] P. Tichavsky and A. Nehorai, "Comparative study of four adaptive frequency trackers," IEEE Trans. Signal Process., vol. 45, no. 6, pp. 14731484, Jun. 1997.

[7] S. Y. Park, Y. S. Song, H. J. Kim, and J. Park, "Improved method for frequency estimation of sampled sinusoidal signals without iteration," IEEE Trans. Instrum. Meas., vol. 60, no. 8, pp. 2828-2834, Aug. 2011.

[8] M. Karimi-Ghartemani, H. Karimi, and M. R. Iravani, "A magnitude/ phase-locked loop system based on estimation of frequency and in-phase/ quadrature-phase amplitudes," IEEE Trans. Ind. Electron., vol. 51, no. 2, pp. 511-517, Apr. 2004

[9] D. Beeman, Industrial Power System Handbook. New York: McGrawHill, 1955.

[10] Y. Xia and D. P. Mandic, "Widely linear adaptive frequency estimation of unbalanced three-phase power systems," IEEE Trans. Instrum. Meas., vol. 61, no. 1, pp. 74-83, Jan. 2012.

[11] D. P. Mandic and V. S. L. Goh, Complex Valued Nonlinear Adaptive Filters: Noncircularity, Widely Linear and Neural Models. Hoboken, NJ: Wiley, 2009.

[12] B. Picinbono and P. Chevalier, "Widely linear estimation with complex data," IEEE Trans. Signal Process., vol. 43, no. 8, pp. 2030-2033, Aug. 1995.

[13] B. Jelfs, D. P. Mandic, and S. C. Douglas, "An adaptive approach for the identification of improper complex signals," Signal Process., vol. 92, no. 2, pp. 335-344, Feb. 2012.

[14] C. Cheong-Took, S. C. Douglas, and D. P. Mandic, "On approximate diagonalization of correlation matrices in widely linear signal processing," IEEE Trans. Signal Process., vol. 60, no. 3, pp. 1469-1473, Mar. 2012.
[15] D. H. Dini and D. P. Mandic, "A class of widely linear complex Kalman filters," IEEE Trans. Neural Netw. Learn. Syst., vol. 23, no. 5, pp. 775786, May 2012.

[16] S. Haykin, Kalman Filtering and Neural Networks. Hoboken, NJ: Wiley, 2001.

[17] S. Sangsuk-Iam and T. E. Bullock, "Behavior of the discrete-time Kalman filter under incorrect noise covariances," in Proc. 26th IEEE Conf. Decision Control, Dec. 1987, vol. 26, pp. 1594-1600.

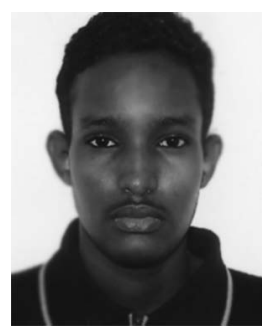

Dahir H. Dini (S'11) received the M.Eng. degree in electrical and electronic engineering from Imperial College London, London, U.K., in 2009, where he is currently working toward the Ph.D. degree in adaptive signal processing.

His current research interests include multidimensional adaptive filtering, nonlinear signal processing, and augmented complex statistics.

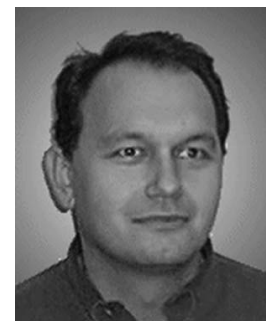

Danilo P. Mandic (M'99-SM'03) received the Ph.D. degree in nonlinear adaptive signal processing from Imperial College London, London, U.K., in 1999 .

$\mathrm{He}$ is a Professor in signal processing at Imperial College London, where he has been working in the area of nonlinear adaptive signal processing and nonlinear dynamics. He has been a Guest Professor at Katholieke Universiteit Leuven, Leuven, Belgium, the Tokyo University of Agriculture and Technology, Tokyo, Japan, and Westminster University, London, and a Frontier Researcher at Riken, Tokyo. His publication record includes two research monographs titled Recurrent Neural Networks for Prediction (West Sussex, U.K.: Wiley, August 2001) and Complex Valued Nonlinear Adaptive Filters: Noncircularity, Widely Linear and Neural Models (West Sussex, U.K.: Wiley, April 2009), an edited book titled Signal Processing for Information Fusion (New York: Springer, 2008), and more than 200 publications on signal and image processing.

Dr. Mandic has been a member of the IEEE Technical Committee on Machine Learning for Signal Processing and an Associate Editor for the IEEE Transactions on CiRcuits AND Systems II, the IEEE TransACTIONS ON Signal Processing, the IEEE Transactions ON NEURAL NETwORKS, and the International Journal of Mathematical Modelling and Algorithms. He has produced award winning papers and products from his collaboration with the industry. He is a member of the London Mathematical Society. 